\title{
Combined associations of body weight and lifestyle factors with all cause and cause specific mortality in men and women: prospective cohort study
}

\author{
Nicola Veronese, ${ }^{1,2}$ Yanping Li, ${ }^{3}$ JoAnn E Manson, 4,5,6 Walter C Willett, ${ }^{3,4,5}$ Luigi Fontana, ${ }^{1,7,8}$ Frank B Hu33,4,5
}

1Division of Geriatrics and Nutritional Science, Washington University, St Louis, MO, USA 2Division of Geriatrics, Department of Medicine, University of Padua, Italy

${ }^{3}$ Department of Nutrition, Harvard T.H. Chan School of

Public Health, Boston, MA, USA ${ }^{4}$ Department of Epidemiology, Harvard T.H. Chan School of Public Health, Boston, MA, USA

${ }^{5}$ Channing Division of Network Medicine, Department of Medicine, Brigham and Women's Hospital, Harvard Medical School, Boston, MA, USA

${ }^{6}$ Division of Preventive Medicine, Department of Medicine, Brigham and Women's Hospital, Boston, MA, USA

${ }^{7}$ Department of Clinical and Experimental Sciences, Brescia University Medical School, Brescia, Italy

${ }^{8}$ CEINGE Biotecnologie Avanzate, Naples, Italy

Correspondence to: Frank B Hu frank.hu@channing.harvard.edu Additional material is published online only. To view please visit the journal online.

Cite this as: BMJ 2016;355:i5855 http://dx.doi.org/10.1136/bmj.i5855

Accepted: 21 October 2016

\section{ABSTRACT}

OBJECTIVE

To evaluate the combined associations of diet, physical activity, moderate alcohol consumption, and smoking with body weight on risk of all cause and cause specific mortality.

DESIGN

Longitudinal study with up to 32 years of follow-up.

SETTING

Nurses' Health Study (1980-2012) and Health

Professionals Follow-up Study (1986-2012).

\section{PARTICIPANTS}

74582 women from the Nurses' Health Study and 39284 men from the Health Professionals Follow-up

Study who were free from cardiovascular disease and cancer at baseline.

\section{MAIN OUTCOME MEASURES}

Exposures included body mass index (BMI), score on the alternate healthy eating index, level of physical activity, smoking habits, and alcohol drinking while outcome was mortality (all cause, cardiovascular, cancer). Cox proportional hazard models were used to calculate the adjusted hazard ratios of all cause, cancer, and cardiovascular mortality with their $95 \%$ confidence intervals across categories of BMI, with 22.5-24.9 as the reference.

\section{RESULTS}

During up to 32 years of follow-up, there were 30013 deaths (including 10808 from cancer and 7189 from cardiovascular disease). In each of the four categories of BMI studied (18.5-22.4, 22.5-24.9, 25-29.9, $\geq 30$ ), people with one or more healthy lifestyle factors had a significantly lower risk of total, cardiovascular, and cancer mortality than individuals with no low risk lifestyle factors. A combination of at least three low risk lifestyle factors and BMI between 18.5-22.4 was

\section{WHAT IS ALREADY KNOWN ON THIS TOPIC}

Leanness is associated with greater metabolic health and a lower risk of type 2

diabetes, cardiovascular disease, cancer, and all cause mortality

Data from some epidemiological studies suggest that being overweight, and

possibly even mildly obese, is associated with a reduced risk of mortality

\section{WHAT THIS STUDY ADDS}

The $U$ shaped relation between BMI and mortality observed in many

epidemiological studies is driven by an over-representation of individuals who are

lean because of chronic metabolic and pathological conditions caused by exposure to smoking, a sedentary lifestyle, and/or unhealthy diets, making them more

susceptible to death

Among overweight and obese individuals, those with one or more low risk lifestyle behaviors have a significantly lower risk of premature mortality

associated with the lowest risk of all cause (hazard ratio $0.39,95 \%$ confidence interval 0.35 to 0.43 ), cancer $(0.40,0.34$ to 0.47$)$, and cardiovascular (0.37, 0.29 to 0.46 ) mortality, compared with those with BMI between 22.5-24.9 and none of the four low risk lifestyle factors.

\section{CONCLUSION}

Although people with a higher BMI can have lower risk of premature mortality if they also have at least one low risk lifestyle factor, the lowest risk of premature mortality is in people in the 18.5-22.4 BMI range with high score on the alternate healthy eating index, high level of physical activity, moderate alcohol drinking, and who do not smoke. It is important to consider diet and lifestyle factors in the evaluation of the association between BMI and mortality.

\section{Introduction}

Data from experimental, epidemiological, and clinical studies indicate that leanness is associated with greater metabolic health and a lower risk of developing type 2 diabetes, cardiovascular disease, and cancer. ${ }^{1-3}$ Data from some epidemiological studies, however, are challenging this concept and suggest that being overweight, and possibly even mildly obese, is associated with a reduced risk of mortality. ${ }^{45}$ Normal weight, defined as a body mass index (BMI) between 18.5-24.9, is a broad reference group containing not only non-smokers who are lean because they are physically active and eating healthy diets but also those who are within the normal BMI range for other reasons that accelerate the ageing process (such as chronic inflammation, chronic obstructive pulmonary disease, and neurodegenerative diseases), probably leading to an artificially reduced mortality among the overweight and moderately obese groups. ${ }^{6-10}$ Therefore, when evaluating the relation between BMI and mortality, it might be important to consider lifestyle factors such as smoking, diet, moderate alcohol intake, and physical activity levels. In addition, the epidemiological studies that reported that overweight and obesity could be associated with reduced mortality have some important limitations. For example, they did not consider diet and lifestyle factors, did not exclude participants with exiting chronic diseases, and had a short duration of follow-up. ${ }^{6}$

Given this background, in this large prospective cohort study, we examined the joint associations of BMI and lifestyle factors with all cause, cardiovascular, and cancer mortality among 74582 women from the Nurses' Health Study (NHS, 1980-2012) and 39284 men from the Health Professionals Follow-up Study (HPFS, 19862012) who were free from cardiovascular disease and 
cancer at baseline and followed up for over 32 years. We hypothesized that individuals who were lean had the lowest risk of mortality, particularly if normal weight was associated with regular exercise, a healthy diet, and no smoking.

\section{Methods}

Study population

The Health Professionals Follow-up Study (HPFS) was established in 1986, when 51529 male US health professionals (dentists, optometrists, osteopaths, podiatrists, pharmacists, and veterinarians) aged 40-75 completed a mailed questionnaire about their medical history and lifestyle. ${ }^{11}$ The Nurses' Health Study (NHS) began in 1976, when 121701 female nurses aged 30-55 responded to a questionnaire regarding medical, lifestyle, and other health related information. ${ }^{11}$ Both cohorts were designed with similar questions on diet, exercise, smoking status, and other factors (the use of hormone replacement therapies and questions related to reproduction were asked only in the nurses' study). In both cohorts, questionnaires have been sent every two years to update this information and identify participants with various newly diagnosed diseases. We excluded participants with previous diagnoses of cancer and cardiovascular diseases at baseline (see table A in appendix 1). We also excluded participants with missing values at baseline for BMI, level of physical activity, score on a food frequency questionnaire, or smoking. After these exclusions, we included 74582 women and 39284 men.

\section{Patient involvement}

No patients were involved in setting the research question or the outcome measures, nor were they involved in the design and implementation of the study. There are no plans to involve patients in dissemination.

\section{Assessment of exposure}

Diet was assessed with a validated food frequency questionnaire that asked how often, on average, a participant had consumed a particular amount of a specific type of food during the previous year. ${ }^{12}$ The intake of nutrients was computed by multiplying the frequency of consumption of each unit of food by its nutrient content, and consumption of alcoholic drinks was also considered. Diet quality was assessed with the score on the alternate healthy eating index (AHEI), which is strongly associated with the onset of metabolic and cardiovascular diseases in the general population. ${ }^{1314}$ Briefly, we assigned points for intake of each component on a scale from 0 to 10, with 10 indicating adherence to the recommended levels of servings per day and 0 the worst intake. Use of multivitamins was scored as 2.5 points for up to four years of use and 7.5 points for five or more years of use. We included 10 components of the index in our diet score: higher intakes of vegetables, fruit, nuts, whole grains, polyunsaturated fatty acids, and long chain omega 3 fatty acids and lower intakes of red and processed meats, sugar sweetened beverages, trans fats, and sodium. In this analysis, scores were divided into fifths, with the two upper fifths classified as healthier diets. ${ }^{15-17}$ The information about diet was updated every four years.

We investigated levels of physical activity using a validated questionnaire, which was updated every two years. ${ }^{18}$ We estimated the hours participants spent each week doing moderate-to-vigorous activities (including brisk walking) that required at least the expenditure of at least 3 MET (metabolic equivalent units) per hour. The validity and reproducibility of the questionnaire have been evaluated and reported previously. Briefly, the correlation between physical activity as reported in one week recalls and that reported on the questionnaires was 0.79 . The correlation between moderate to vigorous activity reported in diaries and that reported on the questionnaires was $0.62 .{ }^{19}$

Smoking habits were categorized as never smoked, former smoker, or current smoker (including the number of cigarettes smoked a day) and updated every two years. Body mass index was calculated as self reported weight $(\mathrm{kg}) /$ height $(\mathrm{m})^{2}$. In our validation study, the correlation between self reported and technician measured weight was $0.97 .^{20}$

\section{Definition of low risk lifestyle group}

Low risk lifestyle factors included never smoking, physical activity levels $\geq 30 \mathrm{~min} /$ day at moderate to vigorous intensity, moderate alcohol consumption defined as 5-15 g alcohol/day in women and 5-30 $\mathrm{g}$ alcohol/day in men, ${ }^{21}$ and the AHEI score in the upper two fifths. Each factor was coded as 0 or 1 , and the sum of these four scores together gave a final score of $0,1,2,3$, or 4 , with higher scores indicating a healthier lifestyle.

\section{Assessment of other confounders}

Information on potential confounders-such as age, ethnicity, use of multivitamins, regular use of aspirin, postmenopausal use of hormones (nurses' study only), and family history of diabetes, cancer, or myocardial infarction (in first degree relatives)-was collected with biennial questionnaires.

\section{Ascertainment of deaths}

We identified deaths from state vital statistics records, the national death index, reports by the families, and the postal system. ${ }^{22}$ The follow-up for death in both cohorts was at least $98 \%$ complete. Cause of death was identified from death certificates or review of medical records. In the current analysis, we evaluated all cause mortality and death from cardiovascular disease (international classification of diseases, eighth revision (ICD-8) codes 390-458) or cancer (ICD-8 codes 140-207).

\section{Statistical analysis}

Participants contributed person time from the return of the baseline questionnaire (1980 for NHS; 1986 for HPFS) until the date of death, loss to follow-up, or the end of the follow-up period (30 June 2012 and 30 January 2012, respectively), whichever came first.

We evaluated the proportional hazards assumption with a likelihood ratio test comparing the model with 
and without an interaction term between time period and the joint categories of BMI and lifestyles. The P value for the proportional hazards assumption was 0.44 , indicating that the assumption was not violated in our analyses. We therefore used Cox proportional hazard models to calculate adjusted hazard ratios for all cause, cancer, and cardiovascular mortality with their 95\% confidence intervals across the categories of BMI (<18.5, 18.5-22.4, 22.5-24.9, 25.0-27.4, 27.5-29.9, 30.0-34.9, 35.0-39.9, and $\geq 40)$. ${ }^{23}$ We defined the BMI range of 22.524.9 as the reference category, as in previous studies. ${ }^{24}$ In our cohorts this was also the range of BMI associated with the lowest mortality (fig A in appendix 2).

We updated the information during follow-up using the most recently available information on BMI and lifestyle factors in which risks of mortality were predicted from the information derived from the most recent questionnaire. For example, in the nurses' study, deaths that occurred from 1 July 1980 to 30 June 1982 were examined in relation to BMI and lifestyle factors based on data collected in the 1980 questionnaire; deaths occurring from 1 July 1982 to 30 June 1984 were examined in relation to BMI and lifestyle factors based on data collected in the 1982 questionnaire, and so forth. For AHEI score, it was updated every four years. The same analytic strategy was applied to HPFS. To minimize potential reverse causality bias, we stopped updating body weight once a participant had a diagnosis of chronic diseases such as cancer, cardiovascular diseases, or diabetes. The covariates included in the multivariable model were age, ethnicity, current use of multivitamins, current use of aspirin, status with regard to a family history of diabetes mellitus, myocardial infarction, or cancer; and, for women, menopausal status and hormone use. If data were missing at a given time point, we carried forward the last observation for one cycle.

We applied two approaches to deal with confounding by smoking: one was based on the whole study population by adjusting for smoking status among people with different BMI categories to minimize the confounding effects of smoking; the other was to analyze only participants who had never smoked.

We calculated the hazard ratios across joint classification of number of low risk lifestyle factors $(0,1,2$, or 23) and BMI (18.5-22.4, 22.5-24.9, 25.29.9, and $\geq 30$ )

\begin{tabular}{lll}
\hline $\begin{array}{l}\text { Table } 1 \mid \text { Age adjusted baseline characteristics of participants in Nurses' Health Study } \\
\text { (NHS, 1980) and Health Professions' Follow-up Study (HPFS, 1986) }\end{array}$ & \\
& NHS, 1980 & HPFS, 1986 \\
No of participants & 74582 & 39284 \\
\hline Mean (SD) age (years) & $45.9(7.2)$ & $52.9(9.5)$ \\
\hline Mean (SD) BMI & $24.3(4.5)$ & $25.5(3.3)$ \\
\hline Mean (SD) score on AHEI & $31.0(6.2)$ & $46.7(10.8)$ \\
\hline Mean (SD) physical activity (hours/week) & $3.9(2.9)$ & $2.8(4.2)$ \\
\hline Mean (SD) alcohol consumption (g/day) & $6.4(10.6)$ & $11.4(15.4)$ \\
\hline Current smoking (\%) & 28.8 & 9.8 \\
\hline High blood pressure (\%) & 15.5 & 19.7 \\
\hline Hypercholesterolemia (\%) & 5.1 & 10.5 \\
\hline Diabetes (\%) & 2.1 & 2.4 \\
\hline BMbboly
\end{tabular}

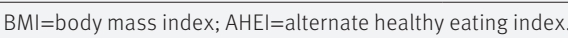

because there were too few cases for cause specific mortality in some groups (see table B in appendix 1). A similar analysis was performed considering only the effect of AHEI, moderate alcohol consumption, and physical activity levels for each BMI category after we excluded participants who had ever smoked.

We ran several sensitivity analyses to test the robustness of our results. First, we kept all missing values at baseline and during follow-up and imputed missing values of BMI and lifestyle factors by applying a multiple imputation approach. Second, we calculated an expanded low risk lifestyle score by assigning weights to each low risk factor based on the $\beta$ coefficients from the multivariable adjusted Cox model with all cause mortality as the outcome. We then summed the products of each binary lifestyle score multiplied by its weight, divided it by the sum of all $\beta$ coefficient values, and then multiplied by 4 to make the low risk lifestyle score easier to interpret. In this way, the expanded low risk lifestyle score ranged from 0 to 4, and each unit of low risk lifestyle score represented the change in one risk factor. Third, we conducted analyses that assessed the effects of specific combination of lifestyle factors on mortality. We used SAS version 9.3 to analyze the data. Significance was set at a two tailed $\mathrm{P}<0.05$.

\section{Results}

At baseline, we included 74582 women from the NHS and 39284 men from the HPFS. Table 1 shows the characteristics of the study population for men and women. Table $C$ in appendix 1 shows their characteristics according to baseline BMI categories. As reported in table D in appendix 1, 8.9\% of women and $19.6 \%$ of men had three or more low risk factors.

During up to 32 years of follow-up, 30013 participants died (10808 from cancer and 7189 from cardiovascular disease). There was a $U$ shaped relation between BMI and mortality among the whole study population (table 2), which was consistent in both women and men. Compared with those with BMI in the 22.5-24.9 range, those in most other BMI categories had an increased risk of all cause mortality after adjustment for potential confounders (table 2). After we excluded people who ever smoked, we found a linear relation between BMI and risk of mortality with the nadir in the 18.5-22.4 BMI range (table 2).

There was a significant interaction between BMI and numbers of low risk lifestyle factors $(\mathrm{P}<0.001$ for interaction, table 3). Among participants without any low risk lifestyle factor, the association between BMI and mortality was $U$ shaped $(\mathrm{P}<0.001$ for non-linear trend, $\mathrm{P}=0.81$ for linear trend). Compared with participants with BMI of 22.5-24.9, those with either BMI $<22.5$ or $>27.5$ had a significantly higher hazard ratio for mortality (table 3 ). Among participants with one or more low risk lifestyle factors the association between BMI and mortality was more linear $(\mathrm{P}<0.001$ for linear trend). Among people with three or four low risk lifestyle factors, compared with those with BMI 22.5-24.9, only participants with BMI $>27.5$, but not those with BMI $<22.5$, had a significantly higher hazard ratio for mortality (table 3). 


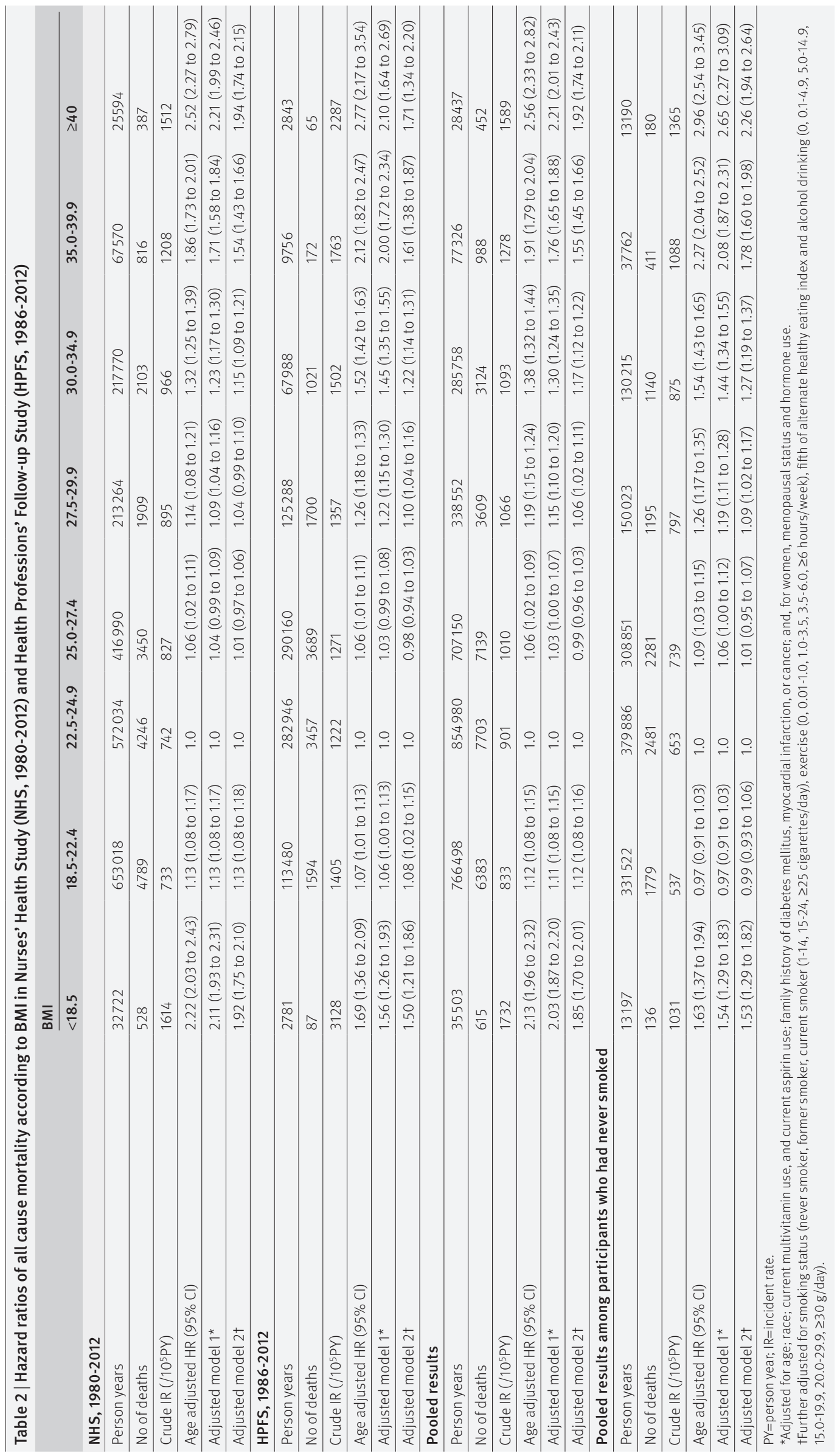




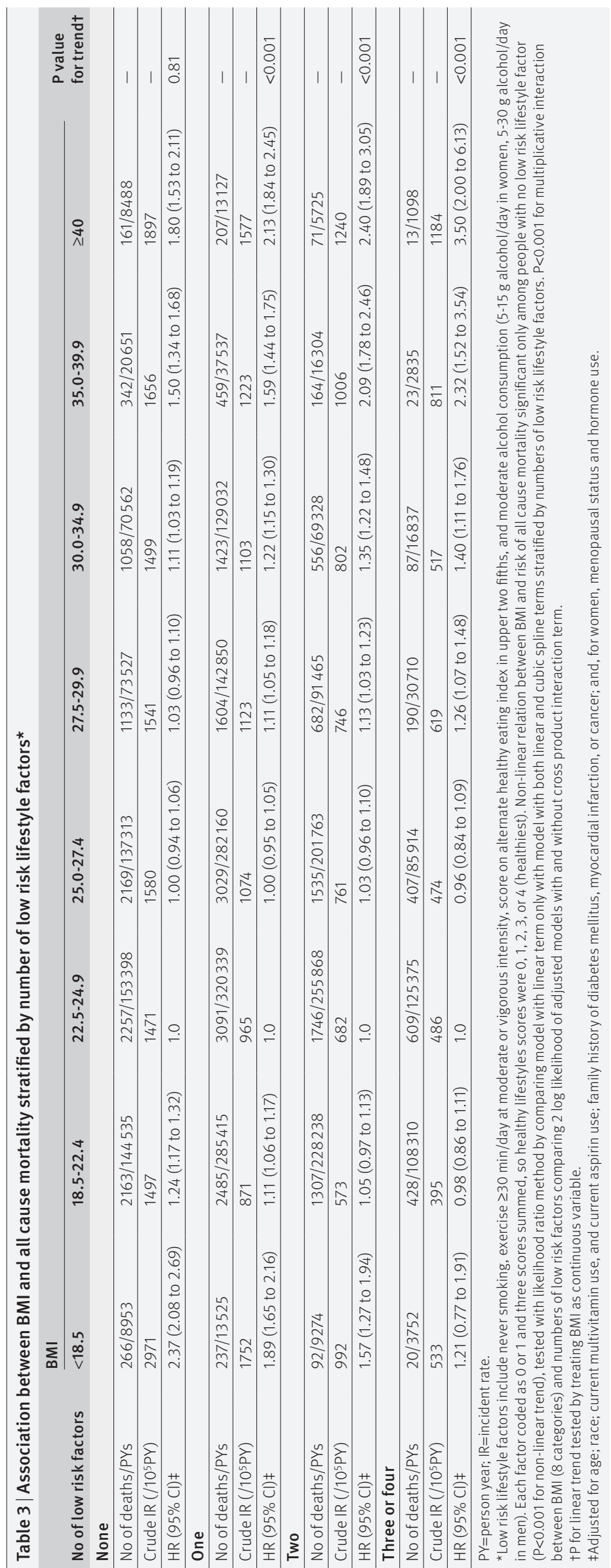

The risk of mortality significantly decreased with the increasing number of low risk lifestyle factors. A combination of at least three low risk lifestyle factors and BMI between 18.5-22.4 was associated with the lowest risk of total (hazard ratio $0.39,95 \%$ confidence interval 0.35 to $0.43)$, cancer (0.40, 0.34 to 0.47$)$, and cardiovascular (0.37, 0.29 to 0.46$)$ mortality, compared with those with BMI between 22.5-24.9 and none of the four low risk lifestyle factors (fig 1). These findings were confirmed in those who had never smoked ( $0.45,0.33$ to 0.61 , for all cause mortality; $0.47,0.29$ to 0.77 , for cancer mortality; $0.41,0.21$ to 0.80 , for cardiovascular disease mortality) (fig 2).

In each of the four BMI categories (18.5-22.4, 22.5-24.9, 25-29.9, $\geq 30$ ), with participants without any low risk factors as the reference, those with one, two, three, or four low risk factors had a graded lower risk for all cause and cause specific mortality (all $\mathrm{P}<0.001$ for trend, fig 1).

The results of sensitivity analyses with multiple imputations (fig B in appendix 2), expanded low risk lifestyle scores (fig $\mathrm{C}$ in appendix 2), or specific combinations of lifestyle factors in the low risk category (fig D in appendix 2) were similar to those from the main analysis.

\section{Discussion}

After adjustment for the effect of high levels of physical activity, healthy diet (AHEI scores), moderate alcohol drinking, and never smoking, men and women with a BMI in the range 18.5-22.4 have a significantly lower risk of cardiovascular disease, cancer, and total mortality. In contrast, men and women with a BMI $<22.4$ and unhealthy lifestyles had a significantly higher risk of mortality than overweight individuals. Our findings suggest that the U shaped relation between BMI and mortality detected in previous epidemiological studies could result from an over-representation of people in our industrialized societies who are, or most likely became, lean because of the chronic accumulation of metabolic, inflammatory, and pathological conditions caused by long term exposure to smoking, a sedentary lifestyle, and unhealthy diets. Interestingly, our data indicate that even in overweight and obese individuals, the exposure to one or more low risk lifestyle behaviors significantly reduced their risk of mortality.

\section{Results in relation to other studies}

Many epidemiological studies have shown a U shaped relation between BMI and mortality, with some studies showing the lowest mortality associated with BMI around 22.5-25 and others around 25-29.9.425 Our data confirm the $\mathrm{U}$ shape relation, with an increased risk associated with BMI $<22.4$ and $\geq 30$, as recently reported in a large meta-analysis of 189 studies without smokers and almost four million participants. ${ }^{26}$ Our data, however, showed for the first time that among men and women who have never smoked with healthier diets, moderate alcohol intake, and regular exercise for $\geq 30 \mathrm{~min} /$ day at moderate intensity, there was a linear relation between BMI and risk of mortality with the nadir in the 18.5-22.4 BMI category. The problem is that few people in North America and Europe are both 

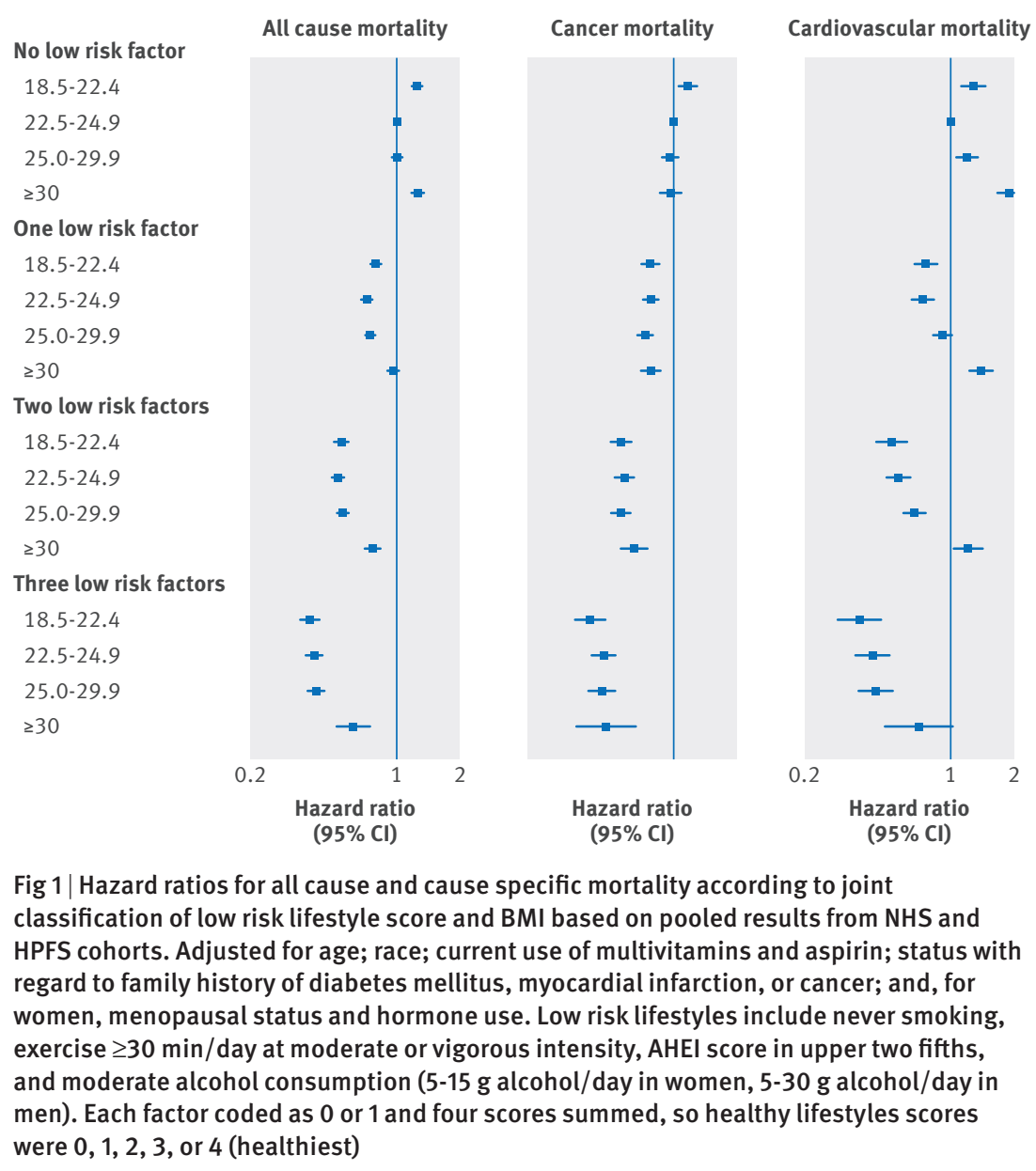

Fig 1 Hazard ratios for all cause and cause specific mortality according to joint classification of low risk lifestyle score and BMI based on pooled results from NHS and HPFS cohorts. Adjusted for age; race; current use of multivitamins and aspirin; status with regard to family history of diabetes mellitus, myocardial infarction, or cancer; and, for women, menopausal status and hormone use. Low risk lifestyles include never smoking, exercise $\geq 30 \mathrm{~min} /$ day at moderate or vigorous intensity, AHEl score in upper two fifths, and moderate alcohol consumption (5-15 g alcohol/day in women, 5-30 g alcohol/day in men). Each factor coded as 0 or 1 and four scores summed, so healthy lifestyles scores were $0,1,2,3$, or 4 (healthiest)

normal weight and practice a healthy lifestyle (that is, not smoking, exercising regularly, moderate alcohol consumption, and eating a healthy diet). ${ }^{27}$ In this study of US health professionals, only $19.6 \%$ of men and $8.9 \%$ of women had three or more low risk lifestyle factors. Our findings suggest that in a substantial proportion of the US population, leanness is driven by other factors that can increase risk of mortality, including existing or preclinical chronic diseases that can cause weight loss and cigarette smoking, which is also associated with lower body weight. The reverse causation problem (that is, low BMI as a consequence of chronic diseases) might be particularly pronounced among those who were sedentary and consuming unhealthy diets, conditions that are important determinants of chronic disease morbidity and mortality. This could explain why we observed an increased mortality in the low BMI range of 18.5-22.4 only among those who did not follow a healthy lifestyle, but not among those with a relatively healthy lifestyle. Increasingly research suggests that smoking, a sedentary lifestyle, and unhealthy hypercaloric diets cause an overstimulation of multiple mitogenic, inflammatory, and oxidative pathways. ${ }^{28-30}$ These factors can accelerate the accumulation of unrepaired molecular damage and senescent cells, stimulate the DNA damage response, inhibit stem cell regenerative function, and promote involuntary weight loss, organ dysfunction, and frailty. ${ }^{70}$ 31-33
Our findings agree with those of a recent meta-analysis, including about 30 million participants, that showed that overweight and obesity are associated with increased risk of all cause mortality, with the nadir of the curve observed at BMI 23-24 among those who had never smoked, 22-23 among healthy people who had never smoked, and 20-22 in studies with a follow-up over 20 years. ${ }^{34}$ In our study, the nadir of the curve was observed at BMI 18.5-22.4 among people who had never smoked and a follow-up period up to 32 years. Of note, in a recent study conducted among three Danish cohorts, the BMI associated with the lowest all cause mortality increased by 3.3 units from cohorts enrolled from 1976-78 to 2003-13. ${ }^{35}$ The average duration of follow-up for the 2003-13 cohort, however, was only 4.6 years, and thus the shift to higher BMI associated with the lowest mortality in this cohort is possibly because of reverse causation bias (that is, individuals with preexisting or preclinical diseases have increased mortality but lower BMI because of weight loss). It should be noted that these studies, unlike ours, did not take into account diet and lifestyle factors.

\section{Potential mechanisms}

BMI is a crude but practical and inexpensive tool to measure adiposity in large population studies. ${ }^{2336}$ It does not, however, measure the ratio of lean to fat or the distribution of fat and, most importantly, the relative contribution of lifestyle and other metabolic factors on the risk of developing a chronic disease and dying prematurely. In this study, we showed that in each of the four categories of BMI (18.5-22.4, 22.5-24.9, 25-29.9, $\geq 30$ ), men and women with healthy lifestyles had significantly lower cardiovascular disease, cancer, and total mortality. Interestingly, even within the obese category, individuals who did not smoke, ate healthier diets, drank a moderate quantity of alcohol, and exercised regularly had a much lower risk of mortality than those who had none of above low risk lifestyle factors. The mechanisms responsible for the protective effects of healthy lifestyles in these individuals are not completely known but probably encompass a series of metabolic and molecular alterations induced by exercise training, healthier diets rich in vitamins and phytochemicals, and avoidance of smoking. These conditions probably inhibit insulin resistance, inflammation, and oxidative stress and slow the accumulation of cellular and organ damage. ${ }^{30-32}$ Our results show that the health benefit of engaging in even one healthy lifestyle behavior is seen in all BMI categories, especially among those in the unhealthy BMI categories.

\section{Strengths and limitations}

Our analysis was based on a large sample size and a long follow-up with a large number of deaths, which provide high statistical power. Another important strength is the high follow-up rate. In each two or four year cycle of the survey of our cohorts, follow-up rates have averaged $94 \%$. In addition, our study includes the repeated measurements of diet, physical activity, smoking, and other factors. Furthermore, we examined not 

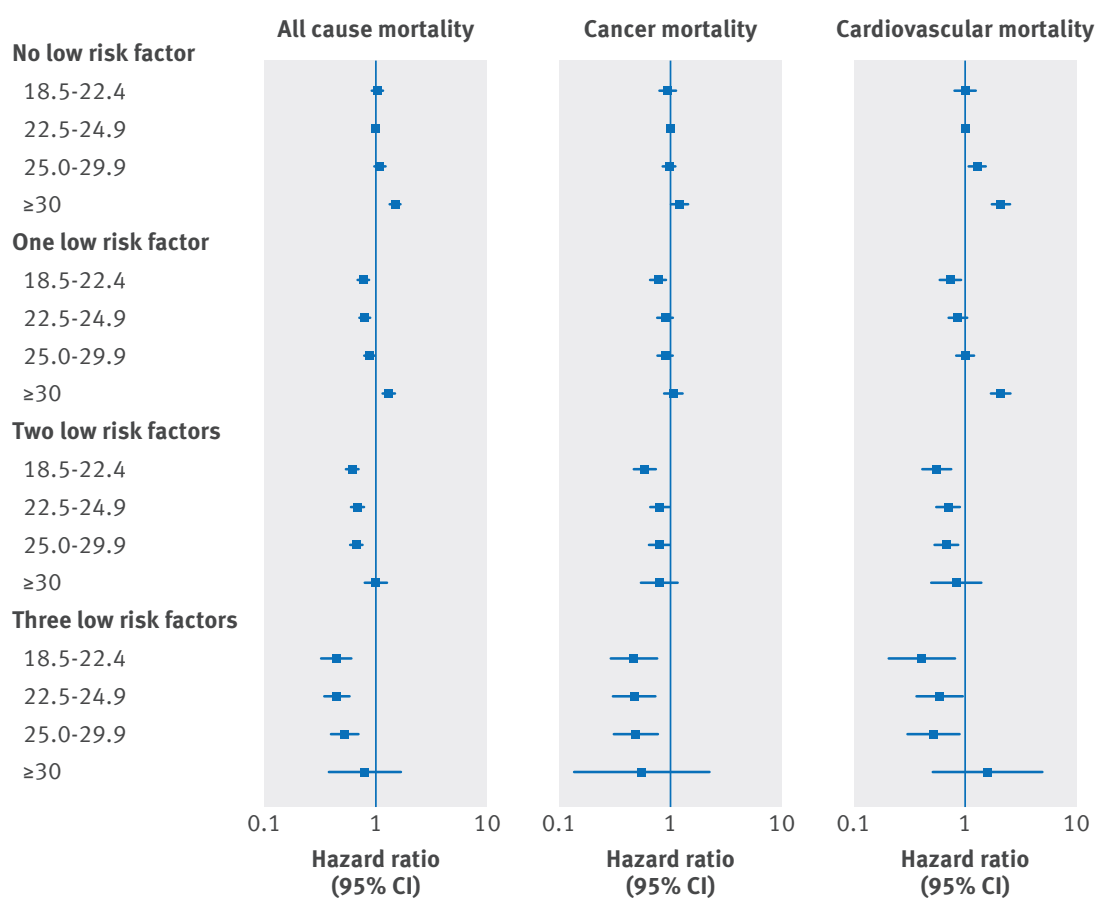

Fig 2 Hazard ratios for all cause and cause specific mortality according to joint classification of low risk lifestyle score and BMI among men and women who had never smoked. Adjusted for age; race; current use of multivitamins and aspirin; status with regard to family history of diabetes mellitus, myocardial infarction, or cancer; and, for women, menopausal status and hormone use. Low risk lifestyles include exercise $\geq 30$ $\mathrm{min} /$ day at moderate or vigorous intensity, alternate healthy eating AHEI score in upper two fifths, and moderate alcohol consumption (5-15 g alcohol/day in women, 5-30 g alcohol/day in men). Each factor coded as 0 or 1 and three scores summed, so healthy lifestyles scores were $0,1,2$, or 3 (healthiest) leanness induced by healthy lifestyles is the optimal way to promote healthy longevity and to reduce the risk of premature death.

We thank the participants and staff of the Nurses' Health Study and the Health Professionals Study who contributed data for their valuable contributions as well as the following state cancer registries for their help: AL, AZ, AR, CA, CO, CT, DE, FL, GA, ID, IL, IN, IA, KY, LA, ME, MD, MA, MI, NE, NH, NJ, NY, NC, ND, OH, OK, OR, PA, RI, SC, TN, TX, VA, WA, WY.

Contributors: LF jointly conceived the study with FBH. NV, YL, LF, and $\mathrm{FBH}$ were involved in the study design. WCW, FBH, and JEM obtained funding. WCW, FBH, and JEM provided study materials or patients and collected and collated data. NV and YL contributed equally to the paper. All authors were involved in analysis and interpretation of the data. NV drafted the article; all authors revised it critically for important intellectual content and approved the final version. LF and $\mathrm{FBH}$ are guarantors.

Funding: The cohorts were supported by grants of UM1 CA186107, P01 CA87969, R01 HL088521, UM1 CA167552, HL60712, and R01 HL35464 from the National Institutes of Health. LF and NV were supported by grants from the Bakewell Foundation and the Longer Life Foundation (an RGA/Washington University Partnership). The funders play no role in the design of the study; the collection, analysis, and interpretation of the data; and the decision to approve publication of the finished manuscript. The authors assume full responsibility for analyses and interpretation of these data.

Competing interests: All authors have completed the ICMJE uniform disclosure form at www.icmje.org/coi_disclosure.pdf (available on request from the corresponding author) and declare: no support from any organization for the submitted work other than those detailed above; no financial relationships with any organizations that might have an interest in the submitted work in the previous three years; no other relationships or activities that could appear to have influenced the submitted work.

Ethical approval: The study protocol was approved by the institutional review boards of the Brigham and Women's Hospital and the Harvard School of Public Health. Completion of the self administered questionnaire was considered to imply informed consent.

Data sharing: No additional data available.

Transparency statement: The lead authors (the manuscript's guarantors) affirm that the manuscript is an honest, accurate, and transparent account of the study being reported; that no important aspects of the study have been omitted; and that any discrepancies from the study as planned (and, if relevant, registered) have been explained.

only the relation between BMI, major lifestyle and all cause mortality but also cause specific mortality.

There are, however, some potential limitations. Our cohorts included only health professionals, mostly white men and women, which might limit the generalizability of the findings. The relative homogeneity of the study population in educational attainment and socioeconomic status, however, enhances the internal validity. Also, though measurement errors in self reported lifestyle factors are inevitable, because of the prospective design these errors are typically unrelated to the outcomes of the study and are thus likely to have attenuated the observed associations.

\section{Conclusions and policy implications}

In conclusion, people in the 18.5-22.4 BMI range with high levels of physical activity, healthy diets, and moderate alcohol consumption and who do not smoke have a substantially lower rate of prematuremortality. One or more low risk lifestyle behaviors also significantly reduce the risk of mortality in overweight and obese individuals. Our data suggest that the U shaped relation between BMI and mortality observed in many epidemiological studies is driven by an over-representation in our societies of individuals who are lean because of chronic metabolic and pathological conditions caused by exposure to smoking, a sedentary lifestyle, and/or unhealthy diets. Finally, our findings indicate that
This is an Open Access article distributed in accordance with the Creative Commons Attribution Non Commercial (CC BY-NC 3.0) license, which permits others to distribute, remix, adapt, build upon this work non-commercially, and license their derivative works on different terms, provided the original work is properly cited and the use is non-commercial. See: http://creativecommons.org/licenses/ by-nc/3.0/.

Fontana L, Hu FB. Optimal body weight for health and longevity: bridging basic, clinical, and population research. Aging Cell 2014;13:391-400. doi:10.1111/acel.12207.

2 National Institutes of Health. Clinical Guidelines on the Identification, Evaluation, and Treatment of Overweight and Obesity in Adults--The Evidence Report. Obes Res 1998;6(Suppl 2):51S-209S. http://www. ncbi.nlm.nih.gov/pubmed/9813653.

3 Rizza W, Veronese N, Fontana L. What are the roles of calorie restriction and diet quality in promoting healthy longevity? Ageing Res Rev 2014;13:38-45. doi:10.1016/j.arr.2013.11.002.

4 Flegal KMK, Kit BK, Orpana H, Graubard BI. Association of all-cause mortality with overweight and obesity using standard body mass index categories: a systematic review and meta-analysis. JAMA 2013;309:71-82. doi:10.1001/jama.2012.113905.

5 Veronese N, Cereda E, Solmi M, et al. Inverse relationship between meta-analysis of 19,538 elderly subjects. Obes Rev 2015;16:1001-15. doi:10.1111/obr.12309.

6 Bhupathiraju SN, Willett WC, Hu FB. Body Mass Index and All-Cause Mortality. JAMA 2016;316:991. doi:10.1001/jama.2016.10282.

7 López-Otín C, Blasco MA, Partridge L, Serrano M, Kroemer G. The hallmarks of aging. Cell 2013;153:1194-217. doi:10.1016/j. cell.2013.05.039.

8 Russell SJ, Kahn CR. Endocrine regulation of ageing. Nat Rev Mol Cell Biol 2007;8:681-91. doi:10.1038/nrm2234.

9 Schumacher B, Garinis GA, Hoeijmakers JHJ. Age to survive: DNA damage and aging. Trends Genet 2008;24:77-85. doi:10.1016/j.tig.2007.11.004. body mass index and mortality in older nursing home residents: a 
10 Fontana L, Partridge L, Longo VD. Extending healthy life span--from yeast to humans. Science 2010;328:321-6. doi:10.1126/ science.1172539.

11 Colditz GA, Rimm EB, Giovannucci E, Stampfer MJ, Rosner B, Willett WC. A prospective study of parental history of myocardial infarction and coronary artery disease in men. Am J Cardiol 1991;67:933-8. http://www.ncbi.nlm.nih.gov/pubmed/2018010. doi:10.1016/0002-9149(91)90163-F.

12 Willett WC, Sampson L, Stampfer MJ, et al. Reproducibility and validity of a semiquantitative food frequency questionnaire. Am J Epidemiol 1985;122:51-65. http://www.ncbi.nlm.nih.gov/pubmed/4014201.

13 Tobias DK, Hu FB, Chavarro J, Rosner B, Mozaffarian D, Zhang C. Healthful dietary patterns and type 2 diabetes mellitus risk among women with a history of gestational diabetes mellitus. Arch Intern Med 2012;172:1566-72. doi:10.1001/archinternmed.2012.3747.

14 McCullough ML, Willett WC. Evaluating adherence to recommended diets in adults: the Alternate Healthy Eating Index. Public Health Nutr 2006;9(1A):152-7. http://www.ncbi.nlm.nih.gov/pubmed/16512963. doi:10.1079/PHN2005938

15 Chiuve SE, Rexrode KM, Spiegelman D, Logroscino G, Manson JE, Rimm EB. Primary prevention of stroke by healthy lifestyle. Circulation 2008;118:947-54. doi:10.1161/CIRCULATIONAHA.108.781062.

16 Hu FB, Manson JE, Stampfer MJ, et al. Diet, lifestyle, and the risk of type 2 diabetes mellitus in women. N Engl J Med 2001;345:790-7. doi:10.1056/NEJMoa010492.

17 Reis JP, Loria CM, Sorlie PD, Park Y, Hollenbeck A, Schatzkin A. Lifestyle factors and risk for new-onset diabetes: a population-based cohort study. Ann Intern Med 2011;155:292-9. doi:10.7326/0003-4819-155-5-201109060-00006

18 Hu FB, Sigal RJ, Rich-Edwards JW, et al. Walking compared with vigorous physical activity and risk of type 2 diabetes in women: a prospective study. JAMA 1999;282:1433-9. http://www.ncbi.nlm.nih. gov/pubmed/10535433. doi:10.1001/jama.282.15.1433.

19 Wolf AM, Hunter DJ, Colditz GA, et al. Reproducibility and validity of a self-administered physical activity questionnaire. Int J Epidemiol 1994;23:991-9. http://www.ncbi.nlm.nih.gov/pubmed/7860180. doi:10.1093/ije/23.5.991.

20 Rimm EB, Stampfer MJ, Colditz GA, Chute CG, Litin LB, Willett WC Validity of self-reported waist and hip circumferences in men and women. Epidemiology 1990;1:466-73. http://www.ncbi.nlm.nih.gov/ pubmed/2090285. doi:10.1097/00001648-199011000-00009.

21 Solomon CG, Hu FB, Stampfer MJ, et al. Moderate alcohol consumption and risk of coronary heart disease among women with type 2 diabetes mellitus. Circulation 2000;102:494-9. doi:10.1161/01.CIR.102.5.494.

22 Rich-Edwards JW, Corsano KA, Stampfer MJ. Test of the National Death Index and Equifax Nationwide Death Search. Am J Epidemio 1994;140:1016-9. http://www.ncbi.nlm.nih.gov/pubmed/7985649.
23 Obesity: preventing and managing the global epidemic. Report of a WHO consultation. World Health Organ Tech Rep Ser 2000;894:i-xii, 1-253. http://www.ncbi.nlm.nih.gov/pubmed/11234459.

24 Berrington de Gonzalez A, Hartge P, Cerhan JR, et al. Body-mass index and mortality among 1.46 million white adults. N Engl J Med 2010;363:2211-9. doi:10.1056/NEJMoa1000367.

25 Tobias DK, Hu FB. Does being overweight really reduce mortality? Obesity (Silver Spring) 2013;21:1746-9. doi:10.1002/oby.20602.

26 Global BMI Mortality Collaboration. Body-mass index and all-cause mortality: individual-participant-data meta-analysis of 239 prospective studies in four continents. Lancet 2016;388:776-86. doi:10.1016/S0140-6736(16)30175-1.

27 Stampfer MJ, Hu FB, Manson JE, Rimm EB, Willett WC. Primary prevention of coronary heart disease in women through diet and lifestyle. N Engl J Med 2000;343:16-22. doi:10.1056/ NEJM200007063430103.

28 Chiolero A, Faeh D, Paccaud F, Cornuz J. Consequences of smoking for body weight, body fat distribution, and insulin resistance. Am J Clin Nutr 2008;87:801-9. http://www.ncbi.nlm.nih.gov/pubmed/18400700.

29 Messner B, Bernhard D. Smoking and cardiovascular disease: mechanisms of endothelial dysfunction and early atherogenesis. Arterioscler Thromb Vasc Biol 2014;34:509-15. doi:10.1161/ ATVBAHA.113.300156.

30 Rom O, Kaisari S, Aizenbud D, Reznick AZ. Sarcopenia and smoking: a possible cellular model of cigarette smoke effects on muscle protein breakdown. Ann N Y Acad Sci 2012;1259:47-53. doi:10.1111/j.1749-6632.2012.06532.x.

31 Booth FW, Roberts CK, Laye M). Lack of exercise is a major cause of chronic diseases. Compr Physiol 2012;2:1143-211. doi:10.1002/cphy. c110025.

32 Campisi J. Aging, cellular senescence, and cancer. Annu Rev Physiol 2013;75:685-705. doi:10.1146/annurev-physiol-030212-183653.

33 Conboy IM, Rando TA. Heterochronic parabiosis for the study of the effects of aging on stem cells and their niches. Cell Cycle 2012;11:2260-7. doi:10.4161/cc.20437.

34 Aune D, Sen A, Prasad M, et al. BMI and all cause mortality: systematic review and non-linear dose-response meta-analysis of 230 cohort studies with 3.74 million deaths among 30.3 million participants. BM 2016;353:i2156. doi:10.1136/bmj.i2156.

35 Afzal S, Tybjærg-Hansen A, Jensen GB, Nordestgaard BG. Change in Body Mass Index Associated With Lowest Mortality in Denmark, 1976-2013. JAMA 2016;315:1989-96. doi:10.1001/jama.2016.4666.

36 Wannamethee SG. Obesity Epidemiology. Frank B Hu. Int J Epidemiol 2009;38:325-6. doi:10.1093/ije/dyn227.

Appendix 1: Supplementary tables A-D

Appendix 2: Supplementary figures A-D 